\title{
Editorial
}

\section{Improving neonatal intubation safety: A journey of a thousand miles}

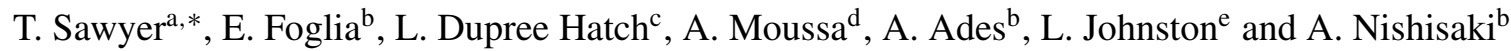 \\ a Seattle Children's Hospital and University of Washington School of Medicine, Department of Pediatric, Division \\ of Neonatology, Seattle, WA, USA \\ ${ }^{\mathrm{b}}$ The Children's Hospital of Philadelphia and The University of Pennsylvania Perelman School of Medicine, \\ Department of Pediatric, Division of Neonatology, Philadelphia, PA, USA \\ ${ }^{\mathrm{c}}$ Vanderbilt University Medical Center and Vanderbilt University School of Medicine, Department of Pediatric, \\ Division of Neonatology, Nashville, TN, USA \\ ${ }^{\mathrm{d}}$ Université de Montréal, Department of Pediatric, Division of Neonatology, Montréal, QC, Canada \\ ${ }^{\mathrm{e}}$ Yale-New Haven Hospital and Yale School of Medicine, Department of Pediatric, Division of Neonatology, \\ New Haven, CT, USA
}

\section{Received 24 July 2016}

Revised 30 November 2016

Accepted 23 January 2017

\begin{abstract}
Neonatal intubation is one of the most common procedures performed by neonatologists, however, the procedure is difficult and high risk. Neonates who endure the procedure often experience adverse events, including bradycardia and severe oxygen desaturations. Because of low first attempt success rates, neonates are often subjected to multiple intubation attempts before the endotracheal tube is successfully placed. These factors conspire to make intubation one of the most dangerous procedures in neonatal medicine. In this commentary we review key elements in the journey to improve neonatal intubation safety. We begin with a review of intubation success rates and complications. Then, we discuss the importance of intubation training. Next, we examine quality improvement efforts and patient safety research to improve neonatal intubation safety. Finally, we evaluate new tools which may improve success rates, and decrease complications during neonatal intubation.
\end{abstract}

Keywords: Endotracheal intubation, tracheal intubation, neonatal intubation, patient safety, quality improvement, intubation associated adverse event

\section{Introduction}

Endotracheal intubation is a fundamental skill that every neonatologist must be competent to perform, however, it is difficult in practice and fraught with risk. Neonates who endure the procedure often experience adverse events, including bradycardia and

\footnotetext{
*Address for correspondence: Taylor Sawyer, DO, Med, University of Washington School of Medicine, 1959 NE Pacific St, RR451 HSB, Box 356320, Seattle, WA 98195 6320, USA. Tel.: +1 206543 3200; Fax: +1 206543 8926; E-mail: tlsawyer@uw.edu.
}

severe oxygen desaturations. Due to low first attempt success rates, neonates are often subjected to a second, third, or even fourth intubation attempt before the endotracheal tube is successfully placed. These factors conspire to make intubation one of the most dangerous procedures in neonatal medicine. Despite the risks, little attention has been paid to neonatal intubation safety.

Improving neonatal intubation safety cannot be accomplished with a single step. As with any patient safety initiative, multiple factors must be considered. 
In this commentary we review some key elements in the journey to improve neonatal intubation safety. We begin with a review of intubation success rates and complications. Then, we discuss the importance of intubation training. Next, we examine quality improvement (QI) interventions and research to improve neonatal intubation safety. Finally, we evaluate new tools which may aid providers in achieving higher success rates, and fewer complications, when performing neonatal intubation.

\section{Neonatal intubation success rates and complications}

How do we define a successful neonatal intubation? Before we can study neonatal intubation success, we must first acknowledge that this outcome has never been adequately defined. Some practitioners consider the insertion of the endotracheal tube into the trachea as success, regardless of the conditions of the intubation. However, the placement of the endotracheal tube into the trachea on the first attempt, without adverse events or complications, may be a better definition of success.

Most available data on neonatal intubation success come from single-site observational studies. These studies have consistently identified experience level and provider discipline as significant factors associated with intubation success (Fig. 1) [1-8]. Experienced providers, such as attending neonatologists, have the highest success rates of around 64\%. Novice providers, such as pediatric residents, have the lowest success rates, with success rates of only 20-26\% [6-8]. Pediatric residents' suboptimal success rates likely stem from the limited experience with neonatal intubation. Gozzo et al. observed that pediatric residents performed few procedures in the NICU [9]. In a 2015 study of pediatric resident neonatal intubation competency, DeMeo et al. cited a median number of 3 intubation opportunities per resident during training [10]. The limited clinical experience is multifactorial, resulting from restrictions in duty hours and NICU rotations [11], increased presence of advanced practice providers in the NICU $[9,12]$, changes in management of infants with meconium-stained amniotic fluid [13], and increasing usage of non-invasive ventilation strategies [14]. As opposed to residents, neonatal fellows report considerably more neonatal intubation experiences, with an average of 60 intubations by graduation [15]. However, the amount of

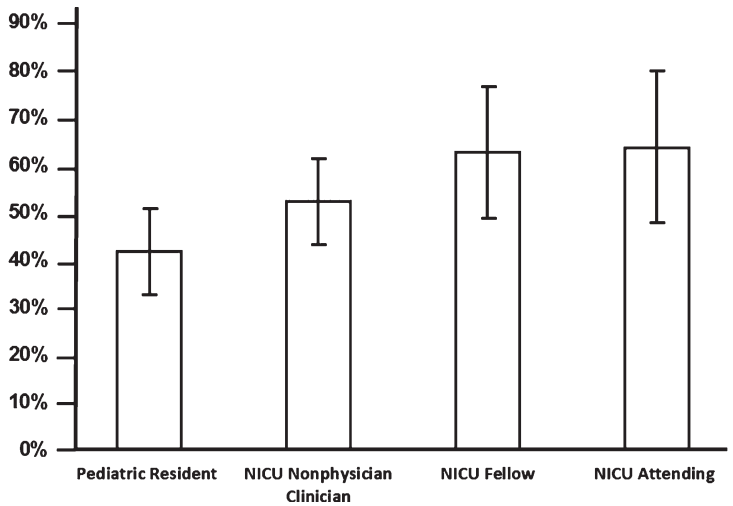

Fig. 1. Neonatal intubation first attempt success rates, presented as mean \pm standard deviation, by provider type based on pooled data from eight published single center and multicenter observational studies (8,066 total intubations) [1-8]. First attempt success rates by provider type are as follows: pediatric residents $42 \pm 9 \%$, NICU non-physician clinicians (nurse practitioners, physician assistants, respiratory therapists and transport nurses) $52 \pm 9 \%$, NICU fellow $63 \pm 14 \%$, NICU attending $64 \pm 16 \%$. The overall first attempt intubation success was $50 \pm 8 \%$.

experience needed to establish competency with the procedure is unknown, and will vary by the individual. This highlights the importance of developing empiric methods to establish procedural competency in trainees [15].

Less is known about the safety of neonatal intubation. Only two studies have focused on neonatal intubation complications and adverse events. Foglia et al. conducted a single-center prospective observational cohort study of infants intubated in a level IV referral NICU. The authors reported an adverse event rate of $22 \%$, with the most common events being esophageal intubation (16\%), mainstem intubation $(2 \%)$, oral/airway trauma $(2.7 \%)$, vomiting $(1.6 \%)$ and cardiac arrest $(0.9 \%)$ [7]. Severe oxygen desaturation, defined as $\geq 20 \%$ decrease in oxygen saturation, occurred in $51 \%$ of encounters [7]. Hatch et al. studied intubations in a level IV academic NICU and reported an adverse event rate of $39 \%$ [8]. The types of adverse events were similar to those reported by Foglia et al., and included esophageal intubation (21\%), oral/airway bleeding $(9.5 \%)$, mainstem intubation $(7 \%)$ and hypotension (3.7\%) [8]. These studies shed light on the high rate of complications associated with neonatal intubation.

The success and safety of neonatal intubation are intrinsically linked. Factors that are associated with improved intubation success, such as attending-level provider and paralytic premedication, were both protective against adverse events in the report by Foglia 
et al. [7] Similarly, Hatch et al. found that the odds of adverse events increased with increasing number of intubation attempts [8]. Thus, interventions to improve provider proficiency at intubation may increase the safety of the procedure.

\section{Neonatal intubation training}

According to the Accreditation Council for Graduate Medical Education, both pediatric residents and NPM fellows must be competent to perform neonatal intubation by the completion of training [11, 16]. As noted above, current pediatric residents have limited experience with neonatal intubation and are unlikely to perform more than a handful of intubations during training. Due to limited clinical opportunities for neonatal intubation and other procedures during pediatric residency, Lopreiato and Sawyer suggested adjunctive simulation-based training [17].

Simulation-based procedural training can be optimized using evidence-based educational practice. The 'Learn-See-Practice-Prove-Do-Maintain' (LSPPDM) training pedagogy is one such approach [18]. Using this method, training in a procedural skill, like neonatal intubation, is divided into 4 phases. In the first phase the trainee is required to learn the procedure through reading, didactic teaching, or e-learning modules. In the second phase, the trainee sees the procedure performed either via direct observation or video review. In the third phase, the trainee deliberately practices the procedure using simulation. In the fourth phase, the trainee proves proficiency with the procedure on a simulator by reaching a pre-defined 'mastery' standard on a validated observational assessment tool. Once initial training and simulationbased assessment are complete, the trainee is then permitted to " $d o$ " the procedure on a patient. During initial attempts, close clinical supervision is required. With increased competency the trainee is allowed to perform the procedure with decreasing levels of supervision through the process of graduated responsibility, or entrustment [19]. Once clinical competency is established, the trainee is allowed to perform the procedure independently, without direct supervision. Procedural competency is maintained through ongoing clinical experience supplemented by simulation-based practice, as needed, when clinical opportunities to perform the procedure are limited.

Neonatal intubation training using the LSPPDM approach has the potential to improve first time success rates, and thus decrease complications. Using a simulation-based mastery learning method, as outlined in the LSPPDM pedagogy, Barsuk et al. were able to improve success rates and decrease complications during central venous catheter placement by residents [20,21]. It is possible that the same results could extend to intubation [22]. Methods to enhance the practice, prove and do phases of neonatal intubation training include innovative technologies such as the use of haptic technology to improve the fidelity of the simulation training, and video laryngoscopy to allow for real-time coaching [23, 24]. Developing ways to maintain neonatal intubation competency for providers who perform the procedure infrequently is important $[25,26]$. Simulation offers the only viable means for such training $[17,18]$. While ensuring provider competency with intubation is critical, technical proficiency alone does not guarantee a successful and safe intubation.

\section{Neonatal intubation safety research}

Performing a successful neonatal intubation is a complex task, involving more than the technical skills of performing the intubation. The factors at play include: provider characteristics, such as competency and experience, practice characteristics, such as the medications and equipment, patient characteristics, such physiologic stability and airway anatomy, and system characteristics, such as the microsystem and safety culture of the unit (Fig. 2). While acquiring the technical skills needed to perform neonatal intubation is critical, building a system to support neonatal intubation safety is equally important. QI methods can be used to build and strengthen these systems.

Hatch et al. described a multi-disciplinary QI project to decrease the incidence of intubation associated adverse events in a large, academic NICU [27]. The project tested three interventions using the Institute for Healthcare Improvements (IHI) Model for Improvement: 1. an "Intubation Timeout" tool to standardize pre-procedural preparation and improve team communication and situational awareness, 2. an evidence-based premedication algorithm for non-emergent intubation, and 3. an intubationspecific computerized provider order entry set. With these interventions adverse events decreased from $46 \%$ (126/273) during the pre-intervention period to $36 \%(85 / 236)$ during the intervention/sustainment period $(\mathrm{RR}=0.78,95 \%$ confidence interval $[\mathrm{CI}]$ $0.63-0.97)$. Bradycardia and hypoxemia significantly 


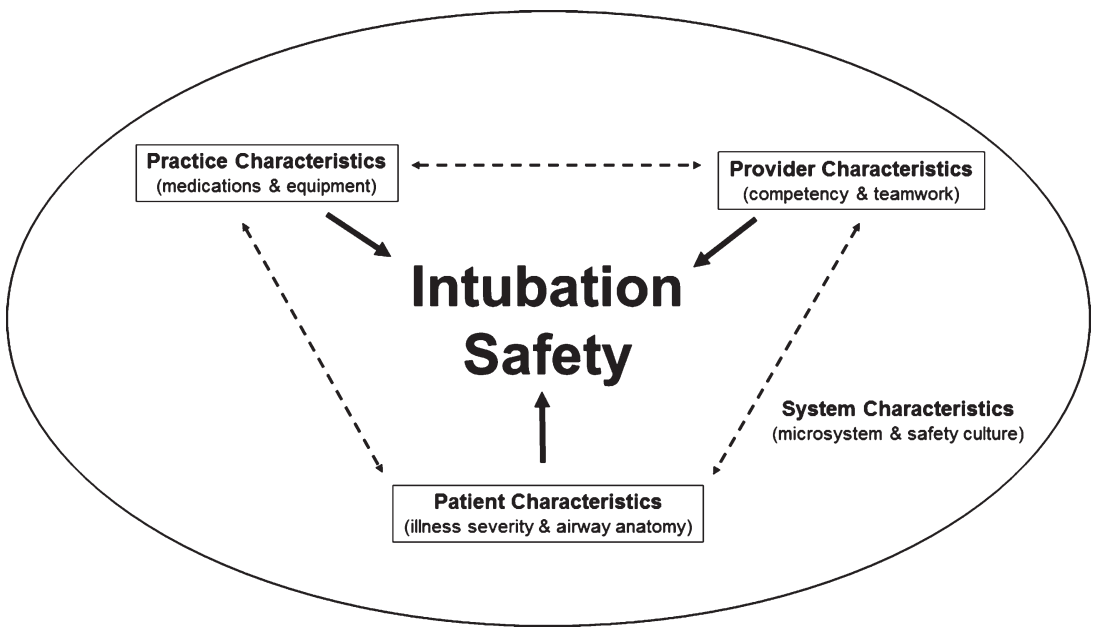

Fig. 2. Factors associated with intubation success and safety.

decreased as well. Using statistical process control methods, the improvements were temporally related to implementation of the "Intubation Timeout" tool [27]. The authors concluded that the improvements noted were due to improvements in team communication and function prior to, and during, the intubation encounter, a finding which has been reported in adult and pediatric intubations as well [28-30].

In addition to QI reports, a growing body of literature exists to inform the safe practice of neonatal intubation. One area of this literature seems especially important - premedication for intubation. Premedication regimens that include neuromuscular blockade have been shown to improve intubation conditions [31], decrease bradycardia and oxygen desaturation [32] and decrease the duration and number of intubation attempts [33, 34]. While supported by the American Academy of Pediatrics [35], premedication is still not widely utilized in many American NICUs [36].

Given the evidence documenting frequent adverse events during neonatal intubation and the paucity of rigorously tested interventions to decrease these events, well-designed multi-center projects are needed to identify and test new interventions to improve airway safety, and to document the contextual and adaptive factors which allow these interventions to be effectively implemented. Future interventions must target those factors shown to be associated with adverse events. These factors include the experience of the intubating clinician, use of muscle relaxants, intubation urgency (emergent vs. non-emergent) and the number of attempts necessary to secure the airway $[7,8]$. Interventions such as the use of premedication with muscle relaxants to decrease the number of intubation attempts, checklists to improve team communication and identification of infants at highest risk of a difficult intubation, and selective criteria for who will perform the intubation will likely improve the safety of this common procedure. The recently formed National Emergency Airway Registry for Neonates (NEAR4NEOS), based at the Children's Hospital of Philadelphia, provides a robust tool for institutions to benchmark tracheal intubation success and adverse event rates, to identify best practices, and to test novel interventions aimed at improving both the success and safety of neonatal intubation. The NEAR4NEOS currently has 12 participating sites and has prospectively collected detailed information on over 2,500 neonatal intubations performed in both the NICU and in the delivery room.

\section{Alternative methods of intubating neonates}

In the 80 years since neonatal intubation was first described the technique most commonly used, direct laryngoscopy, has remained essentially unchanged despite vast improvements in medical technology and equipment [37]. Multiple airway devices have been developed to improve the success and safety of intubation, however, reports of their use in neonates has been limited to case reports [38-40] observational studies [41] and small pilot randomized trials $[42,43]$. Clinical use of some technologies has been limited by the small size of the mouth and airway of the neonate. 
Videolaryngoscopy has shown its clinical usefulness in infants as small as $530 \mathrm{~g}$ [44]. Videolaryngoscopy incorporates a fiberoptic camera lens into the light source of a laryngoscope blade, effectively positioning the laryngoscopist's eye at the tip of the blade, expanding the viewing angle offered by the direct laryngoscope [45]. The videolaryngoscope is connected to a video monitor which displays a magnified image [43]. Video-assisted intubation offers precious teaching opportunities through better identification and recognition of anatomy from the magnified view, and the possibility for both teacher and trainee to share the same visual landmarks allowing guidance of the resident throughout the procedure [43]. Simulation studies have reported improved intubation success rates using videolaryngoscopy $[46,48]$. Recently, two clinical trials have examined the benefits of videolaryngoscope use during neonatal intubation $[49,50]$.

The first study, by O'Shea et al., randomly assigned 206 intubations to be completed by novice pediatric residents using the videolaryngoscope (Laryflex, Acutronics, Hirzel, Switzerland). Each intubation attempt was randomized to either have the screen covered, or visible to a preceptor who could then use the image to coach during the procedure [49]. Thirtysix residents' intubated 168 neonates at a median corrected gestational age of 29 weeks and a median weight of just over $1,100 \mathrm{~g}$. The first-attempt intubation success rate was higher when the screen was visible to the preceptor, compared to when it was covered $(66 \%$ vs. $41 \%, p<0.001)$. The effect was even more significant when patients received premedication $(72 \%$ vs. $44 \%, p<0.001)$. Duration of intubation, lowest oxygen saturation, and lowest heart rate did not differ between study groups.

The second study by Moussa et al. randomly assigned 34 junior pediatric residents to perform endotracheal intubations using either the videolaryngoscope (C-MAC, Karl Storz, Tuttlingen, Germany) or the classic direct laryngoscope [50]. Residents in that study performed 213 intubations on 198 infants at a median corrected gestational age of 32 weeks and a median weight of approximately $1,500 \mathrm{~g}$. Overall intubation success rate was higher with videolaryngoscopy ( $75 \%$ vs. $63 \%, p=0.03)$, and residents reached competency (defined as success rate of over $80 \%$ ) more rapidly with the videolaryngoscope (2nd vs. 7th intubation). Although time to intubation was longer with the videolaryngoscope (57 vs. $47 \mathrm{sec}-$ onds, $p=0.008$ ), this difference was not clinically relevant. There were no differences in number of attempts, number of bradycardia episodes or lowest oxygen saturation between the groups. However, there were more mucosal trauma events with the classic laryngoscope.

Based on the results of these two studies, videolaryngoscopy has the potential to improve success rates for neonatal intubations performed by trainees. Larger scale, multi-center, research is needed to confirm these findings. Research to examine the potential benefits of videolaryngoscopy in more experienced providers is also needed.

\section{Conclusions}

In this commentary we reviewed 4 elements of neonatal intubation; intubation success rates and complications, intubation training, intubation safety research, and the use of videolaryngoscopy. Understanding each of these elements has an important role in making intubation safer for the fragile neonates we care for. Effective training in neonatal intubation requires reliance of evidence-based educational methods. Ensuring adequate procedural experience and tracking competency development in trainees are critical. Conducting QI and clinical research is vital to optimize care and drive practice change. The use of new technology, such as videolaryngoscopy, to improve success rates and lower complications is an important area for further investigation. The journey that neonatology must take to improve neonatal intubation safety is a long one. Luckily, the first steps have been taken. Now the challenge is to continue the journey.

\section{Acknowledgments}

None.

\section{Declaration of interest statement}

The authors have no relevant financial disclosures or conflicts of interest in relation to this work.

\section{References}

[1] Falck AJ, Escobedo MB, Baillargeon JG, Villard LG, Gunkel JH. Proficiency of pediatric residents in performing neonatal endotracheal intubation. Pediatrics 2003;112:1242-7.

[2] Lane B, Finer N, Rich W. Duration of intubation attempts during neonatal resuscitation. J Pediatr 2004;145(1):67-70. 
[3] Leone TA, Rich W, Finer NN. Neonatal intubation: Success of pediatric trainees. J Pediatr 2005;146(5):638-41.

[4] O'Donnell CPF, Kamlin COF, Davis PG, Morley CJ. Endotracheal intubation attempts during neonatal resuscitation: Success rates, duration, and adverse effects. Pediatrics 2006;117(1):e16-21.

[5] Downes KJ, Narendran V, Meinzen-Derr J, McClanahan S, Akinbi HT. The lost art of intubation: Assessing opportunities for residents to perform neonatal intubation. J Perinatol 2012;32(12):927-32.

[6] Haubner LY, Barry JS, Johnston LC, et al. Neonatal intubation performance: Room for improvement in tertiary neonatal intensive care units. Resuscitation 2013; 84(10):1359-64.

[7] Foglia EE, Ades A, Napolitano N, Leffelman J, Nadkarni V, Nishisaki A. Factors associated with adverse events during tracheal intubation in the NICU. Neonatology 2015;108(1):23-9.

[8] Hatch LD, Grubb PH, Lea AS, et al. Endotracheal intubation in neonates: A prospective study of adverse safety events in 162 infants. J Pediatr 2016;168:62-6.

[9] Gozzo YF, Cummings CL, Chapman RL, Bizzarro MJ, Mercurio MR. Who is performing medical procedures in the neonatal intensive care unit? J Perinatol 2011;31(3):206-11.

[10] DeMeo SD, Katakam L, Goldberg RN, Tanaka D. Predicting neonatal intubation competency in trainees. Pediatrics 2015;135(5):e1229-36.

[11] ACGME Program Requirements for Graduate Medical Education in Pediatrics. Effective: July 1, 2013. Available at: https://www.acgme.org/Portals/0/PFAssets/ProgramRequir ements/320_pediatrics_07012015.pdf.

[12] Lee HC, Rhee CJ, Sectish TC, Hintz SR. Changes in attendance at deliveries by pediatric residents 2000 to 2005 . Am J Perinatol 2009;26(2):129-34.

[13] Whitfield JM, Charsha DS, Chiruvolu A. Prevention of meconium aspiration syndrome: An update and the Baylor experience. Proc (Bayl Univ Med Cent) 2009;22(2): 128-31.

[14] DeMauro SB, Millar D, Kirpalani H. Noninvasive respiratory support for neonates. Curr Opin Pediatr 2014; 26(2):157-62.

[15] Sawyer T, French H, Ades A, Johnston L. Neonatalperinatal medicine fellow procedural experience and competency determination: Results of a national survey. J Perinatol 2016;36(7):570-4.

[16] ACGME Program Requirements for Graduate Medical Education in Neonatal-Perinatal Medicine. Effective: July 1, 2013. Available at: https://www.acgme.org/acgme web/portals/0/pfassets/2013-pr-faq-pif/329_neonatal_perin atal_peds_07012013.pdf. Accessed 5/12/16.

[17] Lopreiato JO, Sawyer T. Simulation-based medical education in pediatrics. Acad Pediatr 2015;15(2):134-42.

[18] Sawyer T, White M, Zaveri P, et al. "Learn, See, Practice, Prove, Do, Maintain": An evidence-based pedagogical framework for procedural skill training in medicine. Acad Med 2015;90(8):1025-33.

[19] Ten Cate O, Hart D, Ankel F, et al. Entrustment decision making in clinical training. Acad Med 2016;91(2):191-8.

[20] Barsuk JH, McGaghie WC, Cohen ER, Balachandran JS, Wayne DB. Use of simulation-based mastery learning to improve the quality of central venous catheter placement in a medical intensive care unit. J Hosp Med 2009;4(7): 397-403.

[21] Barsuk JH, McGaghie WC, Cohen ER, O'Leary KJ, Wayne DB. Simulation-based mastery learning reduces complications during central venous catheter insertion in a medical intensive care unit. Crit Care Med 2009;37(10): 2697-701.

[22] McGaghie WC, Issenberg SB, Cohen ER, Barsuk JH, Wayne DB. Medical education featuring mastery learning with deliberate practice can lead to better health for individuals and populations. Acad Med 2011;86(11):e8-9.

[23] Johnston L, Chen R, Whitfill T, Bruno CJ, Levit O, Auerbach M. Do you see what I see? A randomized pilot study to evaluate the effectiveness and efficiency of simulation-based training with videolaryngoscopy for neonatal intubation. BMJ STEL 2015. doi:10.1136/bmjstel-2015-000031.

[24] Agarwal A, Leviter J, Mannarino C, Levit O, Johnston L, Auerbach M. Is a haptic simulation interface more effective than computer mouse-based interface for neonatal intubation skills training? BMJ STEL 2015. doi:10.1136/bmjstel-2015-000016.

[25] Feigin RD, Drutz JE, Smith EO, Collins CR. Practice variations by population: Training significance. Pediatrics 1996;98:186-92.

[26] Wood AM, Jones MD Jr, Wood JH, Pan Z, Parker TA. Neonatal resuscitation skills among pediatricians and family physicians: Is residency training preparing for post residency practice? J Grad Med Educ 2011;3(4):475-80.

[27] Hatch LD, Grubb PH, Lea AS, et al. Interventions to improve patient safety during intubation in the neonatal intensive care unit. Pediatrics 2016; In Press.

[28] Kerrey BT, Mittiga MR, Rinderknecht AS, et al. Reducing the incidence of oxyhaemoglobin desaturation during rapid sequence intubation in a paediatric emergency department. BMJ Qual Saf 2015;24(11):709-17.

[29] Mayo PH, Hegde A, Eisen LA, Kory P, Doelken P. A program to improve the quality of emergency endotracheal intubation. J Intensive Care Med 2011;26(1):50-6.

[30] Smith KA, High K, Collins SP, Self WH. A preprocedural checklist improves the safety of emergency department intubation of trauma patients. Acad Emerg Med 2015;22(8):989-92.

[31] Lemyre B, Cheng R, Gaboury I. Atropine, fentanyl and succinylcholine for non-urgent intubations in newborns. Arch Dis Child Fetal Neonatal Ed 2009;94(6):F439-42.

[32] Roberts KD, Leone TA, Edwards WH, Rich WD, Finer NN. Premedication for nonemergent neonatal intubations: A randomized, controlled trial comparing atropine and fentanyl to atropine, fentanyl, and mivacurium. Pediatrics 2006;118(4):1583-91.

[33] Le CN, Garey DM, Leone TA, Goodmar JK, Rich W, Finer NN. Impact of premedication on neonatal intubations by pediatric and neonatal trainees. J Perinatol 2014;34(6):45860.

[34] Dempsey EM, Al Hazzani F, Faucher D, Barrington KJ. Facilitation of neonatal endotracheal intubation with mivacurium and fentanyl in the neonatal intensive care unit. Arch Dis Child Fetal Neonatal Ed 2006;91(4):F279-82.

[35] Kumar P, Denson SE, Mancuso TJ, et al. Premedication for nonemergency endotracheal intubation in the neonate. Pediatrics 2010;125(3):608-15.

[36] Muniraman HK, Yaari J, Hand I. Premedication use before nonemergent intubation in the newborn infant. Am J Perinatol 2015;32(9):821-24.

[37] Flagg PJ. Treatment of asphyxia in the newborn. Preliminary report of the practical application of modern scientific methods. JAMA 1928;91:788-91.

[38] Holm-Knudsen R. The difficult pediatric airway-a review of new devices for indirect laryngoscopy in children younger than two years of age. Paediatr Anaesth 2011;21(2):98-103. 
[39] Lafrikh A, Didier A, Bordes M, Semjen F, NouetteGaulain K. Two consecutive intubations using neonatal Airtraq in an infant with difficult airway. Annales Francaises d'Anesthesie et de Reanimation 2010;29(3):245-6.

[40] Hirabayashi Y, Otsuka Y. Early clinical experience with GlideScope video laryngoscope in 20 infants. Paediatr Anaesth 2009;19(8):802-4.

[41] Mutlak H, Rolle U, Rosskopf W, et al. Comparison of the TruView infant EVO2 PCD and C-MAC video laryngoscopes with direct Macintosh laryngoscopy for routine tracheal intubation in infants with normal airways. Clinics (Sao Paulo) 2014;69(1):23-7.

[42] Singh R, Singh P, Vajifdar H. A comparison of Truview infant EVO2 laryngoscope with the Miller blade in neonates and infants. Paediatr Anaesth 2009;19(4):338-42.

[43] Fiadjoe JE, Kovatsis P. Videolaryngoscopes in pediatric anesthesia: What's new? Minerva Anestesiol 2014;80(1):76-82.

[44] Vanderhal AL, Berci G, Simmons CF, Hagiike M. A videolaryngoscopy technique for the intubation of the newborn: Preliminary report. Pediatrics 2009;124(2):e339-e46.
[45] Vlatten A, Aucoin S, Litz S, et al. A comparison of the STORZ video laryngoscope and standard direct laryngoscopy for intubation in the Pediatric airway - a randomized clinical trial. Paediatr Anaesth 2009;19(11):1102-7.

[46] Vanderhal AL, Berci G, Simmons CF. Video assisted endotracheal intubation: Role in teaching and acquiring skills in era of decreasing DR and NICU time during residency training. E-PAS2007:61790722007.

[47] Donoghue AJ, Ades AM, Nishisaki A, Deutsch ES. Videolaryngoscopy versus direct laryngoscopy in simulated pediatric intubation. Ann Emerg Med 2013;61(3):271-7.

[48] Assaad MA, Lachance C, Moussa A. Learning neonatal intubation using the videolaryngoscope: A randomized trial on mannequins. Simul Healthc 2016;11(3):190-3.

[49] O'Shea JE, Thio M, Kamlin CO, et al. Videolaryngoscopy to teach neonatal intubation: A randomized trial. Pediatrics 2015;136(5):912-9.

[50] Moussa A, Luangxay Y, Tremblay S, et al. Videolaryngoscope for teaching neonatal endotracheal intubation: A randomized controlled trial. Pediatrics 2016;137(3):1-8. 